\title{
Incidence of Oxaliplatin Hypersensitivity Reaction Among Colorectal Cancer Patients: a 5-Year Retrospective Study
}

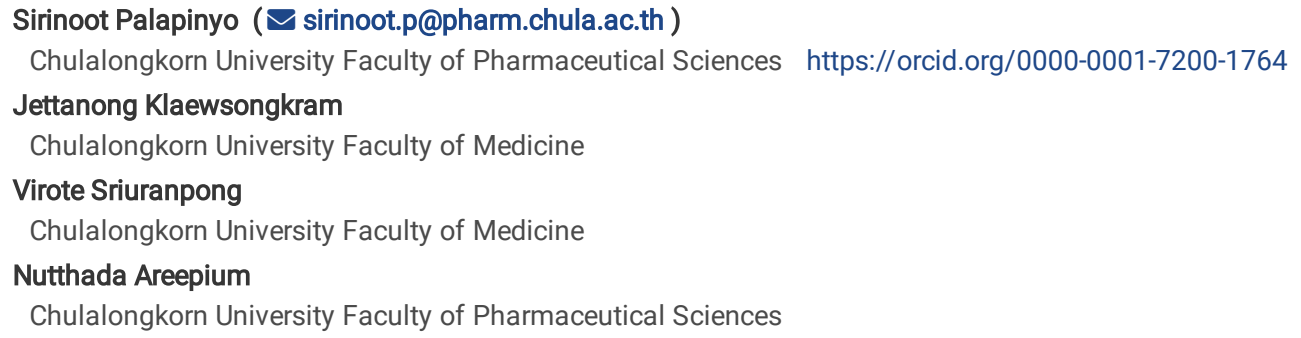

\section{Research Article}

Keywords: oxaliplatin, hypersensitivity reactions, colorectal cancer, risk factors

Posted Date: January 3rd, 2022

DOI: https://doi.org/10.21203/rs.3.rs-1102565/v1

License: () (1) This work is licensed under a Creative Commons Attribution 4.0 International License. Read Full License 


\section{Abstract}

Purpose

We explored the clinical data of colorectal cancer patients treated with oxaliplatin-based regimen to determine the incidence, severity, and risk factors of oxaliplatin-related hypersensitivity reaction (HSR).

\section{Method}

This retrospective study investigated 245 colorectal cancer patients (1,690 treatment cycles) receiving care at King Chulalongkorn Memorial Hospital, Thai Red Cross society between January 1, 2015, and December 31, 2019. The patients' demographic data, laboratory data, and clinical features suggesting hypersensitivity reactions to oxaliplatin were reviewed. The Fisher's Exact test and unpaired t-test were used to determine the differences among patients with and without oxaliplatin HSR. The potential risk factors for oxaliplatin HSR were analyzed for statistical significance by logistic regression.

\section{Results}

A total of 245 colorectal cancer patients (1,690 treatment cycles) were included in this study. The incidence of oxaliplatin HSR was $37.96 \%$, according to the NCl-CTCAE v.5, (grade 1, grade 2, and higher grades were 27.35\% (67 patients), 6.53\% (16 patients), and $4.08 \%$ (10 patients), respectively). The proportion of male patients and patients with history of prior exposure to platinum-based chemotherapy were statistically higher in the HSR group. The eosinophil count and serum creatinine level were also significantly greater in the HSR group. On the contrary, the total lymphocyte count and serum albumin level were significantly lower in the HSR group. The multivariate logistic regression found 5 risk factors with significant difference. Male gender, prior exposure to platinum-based chemotherapy, and elevated eosinophil count were associated with increased risk of oxaliplatin HSR, whereas elevated monocyte count and elevated serum albumin were protective factors for the development of oxaliplatin HSR.

\section{Conclusion}

Colorectal cancer patients treated with oxaliplatin-based regimen with male gender, prior exposure to platinum-based chemotherapy, and elevated eosinophil count have a greater risk of oxaliplatin related hypersensitivity reactions.

\section{Introduction}

Colorectal cancer (CRC) is the fourth most common cancer in the world and the second leading cause of cancer death in the world. CRC treatment is presently a public health priority and oxaliplatin represents a key chemotherapeutic agent.[2, 22]

Oxaliplatin is a third-generation platinum compound with a 1,2-diaminocyclohexane carrier ligand, has been approved both for treatment of metastatic colorectal cancer and adjuvant treatment in combination with 5-fluorouracil (5-FU) and leucovorin (LV) or capecitabine.[8,23] The adverse reactions of oxaliplatin include nausea, vomiting, diarrhea, neutropenia, thrombocytopenia, peripheral neuropathy, and hypersensitivity reaction (HSR).[13, 20] HSR to oxaliplatin has been less frequently described than cisplatin or carboplatin. At the first use, the incidence of HSR to oxaliplatin was very rare, but after expanded use of oxaliplatin in clinical practice, we are now encountering a significant increase of oxaliplatin-HSR. Recent reports have shown that the rate of HSR to oxaliplatin has varied from $8.9-23.8 \% .[7,11,18,28]$ HSR to oxaliplatin should be of concern, due to its unpredictability, it is potentially life-threatening and the subsequent treatment withdrawal. Identifying patients with risk of oxaliplatin-HSR is a key clinical issue and several studies have shown supporting risk factors, with various results. $[11,16,26,29]$ In this retrospective study, we explored the incidence, severity, and risk factors of oxaliplatin-HSR.

\section{Method}

We conducted a retrospective review of the medical records of colorectal cancer patients treated with oxaliplatin-based regimen at King Chulalongkorn Memorial Hospital, Thai Red Cross society between January 1, 2015, and December 31, 2019. All patients received intravenous dexamethasone 8 mg and ondansetron $8 \mathrm{mg}$ as premedication. HSR was assessed and classified according to the National Cancer Institute Common Criteria (NCl-CTCAE v5.0)[6] and was noted by attending healthcare in the medical records. The following data were collected: gender, age, type of cancer, treatment regimen, oxaliplatin dose administration, the baseline laboratory data, the number of cycles administered when the episode occurred, and the severity of HSR. In addition, the management, the response to treatment, the mode of prevention used during rechallenging and the final decision (oxaliplatin continuation or withdrawal) were reviewed. This study was approved by the Institutional Review Board (IRB), Faculty of Medicine, Chulalongkorn University (IRB no. 323/61).

\section{Data analysis}

Statistical analysis was performed using the SPSS software, version 22 (IBM Corp., Armonk, NY, USA). After collecting the data, we imputed the missing data by using multiple regression. Quantitative data are reported as means and standard deviation, and qualitative data are shown as numbers and percentage. Incidence was defined as the number of cases divided by the total number of patients included in the study. The correlations between HSR to oxaliplatin and several background factors were statistically analyzed using Fisher's exact test or the unpaired t-test. In statistical testing, two-sided $p$-values $\leq 0.05$ were considered statistically significant.

The risk factors examined included gender, age, body mass index, ECOG performance status, preexisting allergies (allergy for specific food or drug) and preexposure to platinum chemotherapy. The results from laboratory test data were obtained for 1 month prior to the day of administration of oxaliplatin regimen, which were also analyzed, including serum creatinine, serum glutamic-oxaloacetic transaminase (SGOT), serum glutamate-pyruvate transaminase (SGPT), 
alkaline phosphatase (ALP), serum albumin, white blood cell (WBC) count, neutrophil count, lymphocyte count, monocyte count, and eosinophil count. To determine risk factors potentially associated with HSR to oxaliplatin, those factors were collected and subjected to univariate and multivariate logistic regression. All variables with a $p$-values $<0.2$ were included in the initial multivariate model. Multivariate analysis with backward stepwise elimination was then conducted to develop the final model. Goodness of fit for each stepwise model was compared with Hosmer and Lemeshow test. A $p$-value of $<0.05$ was considered significant.

\section{Results}

We retrospectively analyzed the records of 245 colorectal cancer patients (1,690 treatment cycles) who treated with oxaliplatin-based regimens. The median age was 61 years (range 33-88 years), and 142 (57.96\%) were male. Approximately $70 \%$ of patients had received capecitabine plus oxaliplatin regimen (CapeOx). The background characteristics of colorectal cancer patients who had positive and negative experience of oxaliplatin-HSR and the baseline laboratory data were listed in Table 1. 
Table 1

Patient characteristics $(n=245)$

\begin{tabular}{|c|c|c|c|}
\hline \multirow[t]{3}{*}{ Parameter } & \multicolumn{2}{|l|}{ Number of patients (\%) } & \multirow[t]{3}{*}{$p$-value } \\
\hline & Patient without HSR $(n=152)$ & Patient with HSR ( $n=93)$ & \\
\hline & [Max,Min] & [Max,Min] & \\
\hline Age (years), mean \pm SD & $59.34 \pm 11.38[81,33]$ & $59.96 \pm 11.19[88,40]$ & 0.677 \\
\hline Gender: Male/Female & $79(52.0) / 73(48.0)$ & $63(67.7) / 30(32.3)$ & $0.015^{\star}$ \\
\hline Body surface area $\left(\mathrm{m}^{2}\right)$, mean $\pm \mathrm{SD}$ & $1.62 \pm 0.19[2.23,1.12]$ & $1.64 \pm 0.15[2.01,1.27]$ & 0.370 \\
\hline \multicolumn{4}{|l|}{ ECOG Performance Status } \\
\hline 0 & $1(0.7)$ & $0(0.00)$ & 0.574 \\
\hline 1 & $133(87.5)$ & $79(84.9)$ & \\
\hline 2 & $18(11.8)$ & $14(15.1)$ & \\
\hline Metastasis, yes & $88(57.9)$ & $46(49.5)$ & 0.198 \\
\hline Prior exposure to platinum-based chemotherapy, yes & $18(11.8)$ & $23(24.7)$ & $0.009 *$ \\
\hline History of drug hypersensitivity, yes & $13(5.3)$ & $12(4.9)$ & 0.275 \\
\hline Underlying medical condition, yes & $97(63.8)$ & $55(59.1)$ & 0.499 \\
\hline \multicolumn{4}{|l|}{ Regimen } \\
\hline mFOLFLOX & $15(9.9)$ & $16(17.2)$ & 0.059 \\
\hline CapeOx & $104(68.4)$ & $66(71.0)$ & \\
\hline FLOX & $33(11.8)$ & $11(11.8)$ & \\
\hline \multicolumn{4}{|l|}{ Purpose } \\
\hline Adjuvant & $81(53.3)$ & $70(75.3)$ & $0.001^{*}$ \\
\hline Palliative & $71(46.7)$ & $23(24.7)$ & \\
\hline Dose $(\mathrm{mg})$, mean $\pm \mathrm{SD}$ & $177.36 \pm 45.78[250,65]$ & $183.06 \pm 37.51[250,100]$ & 0.290 \\
\hline \multirow[t]{2}{*}{ Dose per body surface area $\left(\mathrm{mg} / \mathrm{m}^{2}\right)$, mean $\pm \mathrm{SD}$} & $108.71 \pm 23.70$ & $111.58 \pm 21.55[142.86,55.87]$ & 0.342 \\
\hline & {$[135.80,43.62]$} & & \\
\hline \multirow[t]{2}{*}{ Cumulative dose $(\mathrm{mg})$, mean $\pm \mathrm{SD}$} & $1160.53 \pm 471.58$ & $1234.11 \pm 465.85[2400,180]$ & 0.235 \\
\hline & {$[2040,125]$} & & \\
\hline Total infusion course, median & $8[12,1]$ & $7[12,1]$ & $0.844^{b}$ \\
\hline WBC count $\left(x 10^{3} / \mu \mathrm{L}\right)$ & $6.95 \pm 2.26[14.74,2.19]$ & $6.78 \pm 2.05[14.07,3.99]$ & 0.329 \\
\hline \multirow[t]{2}{*}{ Neutrophil count (cells/mm3) } & $4468.09 \pm 1922.92$ & $4414.39 \pm 1764.801[11017,1828]$ & 0.827 \\
\hline & {$[12087,1473]$} & & \\
\hline \multirow[t]{2}{*}{ Total lymphocyte count (cell/mm³) } & $1690.83 \pm 914.56$ & $1459.30 \pm 639.54$ & $0.033^{*}$ \\
\hline & {$[6222,131]$} & {$[3185,356]$} & \\
\hline
\end{tabular}

$* P<0: 05,{ }^{a}$ Fisher's Exact Test
mFOLFLOX: oxaliplatin plus leucovorin and 5-Fluorouracil every 2 weeks
XELOX: Intravenous oxaliplatin $130 \mathrm{mg} / \mathrm{m}^{2}$ (day 1 ) followed by oral capecitabine $1,000 \mathrm{mg} / \mathrm{m}^{2}$ twice daily (day 1 , evening, to day 15 , morning)
WBC white blood cell
FLOX: 5-Fluorouracil plus oxaliplatin on weeks 1,3,5 of 8-week cycle
SGOT: Serum glutamic-oxaloacetic transaminase
SGPT: Serum glutamate-pyruvate transaminase
eGFR: estimated glomerular filtration rate




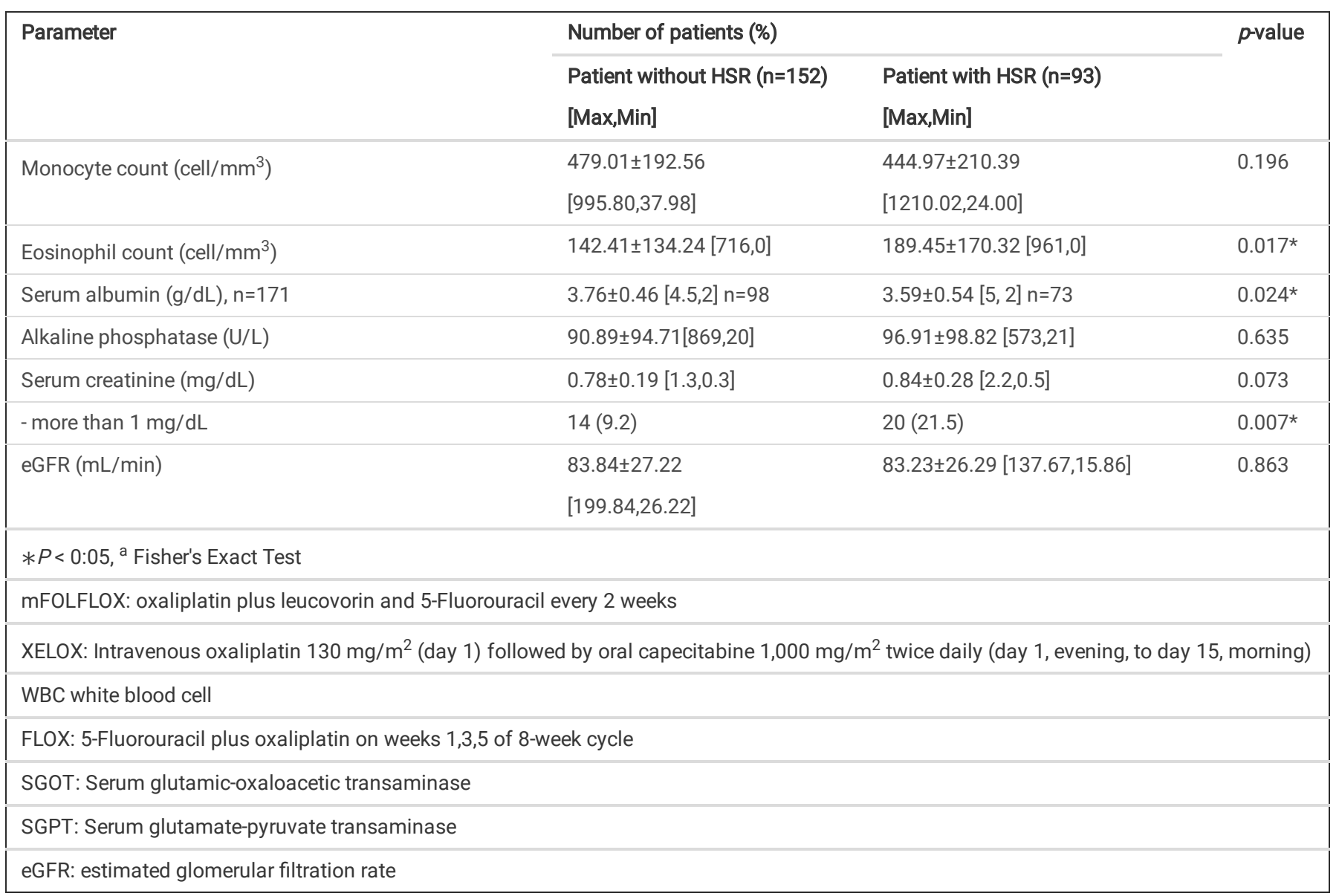

There were no statistical differences of age, body surface area, dosage regimen, performance status, total infusion course, and a history of drug hypersensitivity between the patients without HSR (control group) and with HSR (case group). Compared with women, men had a higher susceptibility to HSR $(p=0.015)$. Those patients who had been exposed to platinum compound prior to this episode of treatment also had higher HSR reports $(p=0.009)$. Baseline biochemical examination of blood showed that the percentage of lymphocytes, eosinophiles and serum albumin were statistically different between the 2 groups ( $p=0.044,0.012,0.024$ respectively).

\section{Incidences of hypersensitivity reaction}

During the 5-year follow-up, 93 patients experienced oxaliplatin-HSR. According to NCI-CTCAE v.5, the incidence rate of grade 1, grade 2 and higher grade was 27.3\% (67 patients), 6.5\% (16 patients) and 4.1\% (10 patients), respectively. (Table 2) Oxaliplatin hypersensitivity appeared on a median of 3 infusions (1-11). In addition, out of 23 patients (9.39\%) who developed oxaliplatin-HSR at first and second infusion course, 10 (43.48\%) had been exposed to platinum agents prior to this episode of treatment. The most common HSR were mild cutaneous reactions such as flushing, urticaria, itching and small area erythema, largely on palms and soles. Some patients presented more severe reactions with facial swelling, dyspnea, wheezing, stomach cramp, diarrhea, or changes of blood pressure. (Table 2) Eleven patients were diagnosed anaphylaxis, with acute and severe symptoms during the infusion or started within 30 minutes after complete infusion. Ten of these were grade 3 HSR, in which the oxaliplatin infusion was immediately discontinued when the reaction occurred and was promptly handled before being admitted into the hospital. (Table 3) 
Table 2

Severity and manifestations of oxaliplatin hypersensitivity reactions $(n=245)$

\begin{tabular}{|ll|}
\hline & Number of patients (\%) \\
\hline Incidence of hypersensitivity & $93(37.96)$ \\
\hline Severity & $67(27.35)$ \\
\hline Grade 1 & $16(6.53)$ \\
\hline Grade 2 & $10(4.08)$ \\
\hline Grade 3/4 & \\
\hline Symptom & $58(23.67)$ \\
\hline Cutaneous reactions & $53(21.63)$ \\
\hline Peripheral neuropathy & $11(4.49)$ \\
\hline Anaphylaxis & $20(8.16)$ \\
\hline Digestive symptoms & $17(6.94)$ \\
\hline Respiratory symptoms & $6(2.45)$ \\
\hline Blood pressure rising & $4(1.63) / 6(2.45)$ \\
\hline Fever/Chill & $3(1.22)$ \\
\hline Palpitation & $2(0.82)$ \\
\hline Blood pressure lowering & $3(1-11)$ \\
\hline Cycle number at event, median (range) & $4(1-11)$ \\
\hline Grade 2, median (range) & $2.5(1-6)$ \\
\hline Grade 3, median (range) & \\
\hline
\end{tabular}


Table 3

Characteristics of the ten patients with grade $3 / 4$ oxaliplatin hypersensitivity

\begin{tabular}{|c|c|c|c|c|c|c|c|c|c|c|c|c|}
\hline No. & Gender & $\begin{array}{l}\text { Age } \\
\text { (years) }\end{array}$ & $\begin{array}{l}\text { Prior } \\
\text { platinum } \\
\text { exposure }\end{array}$ & Metastasis & Regimen & Purpose & $\begin{array}{l}\text { Dose per } \\
\text { BSA } \\
(\mathrm{mg} / \mathrm{m} 2)\end{array}$ & $\begin{array}{l}\text { Cycle } \\
\text { number } \\
\text { at } \\
\text { event }\end{array}$ & $\begin{array}{l}\text { Onset of } \\
\text { HSR } \\
\text { (minutes) }\end{array}$ & $\begin{array}{l}\text { Total } \\
\text { cycle }\end{array}$ & Treatment & Managem \\
\hline \multirow[t]{2}{*}{1} & $\mathrm{~F}$ & 59 & $\mathrm{~N}$ & $\mathrm{~N}$ & CapeOx & A & 130 & 5 & 50 & 5 & $\begin{array}{l}\text { 1. CPM } 10 \mathrm{mg} \text {, } \\
\text { Dexamethasone } \\
5 \text { mg IV STAT }\end{array}$ & \multirow{2}{*}{$\begin{array}{l}\text { Discontin } \\
\text { and adjus } \\
\text { single } \\
\text { capecitabi } \\
\text { then re- } \\
\text { evaluation }\end{array}$} \\
\hline & & & & & & & & & & & $\begin{array}{l}\text { 2. Hold } 30 \\
\text { minutes then } \\
\text { slow infusion } \\
\text { about } 6 \text { hours }\end{array}$ & \\
\hline \multirow[t]{5}{*}{2} & \multirow[t]{5}{*}{ M } & \multirow[t]{5}{*}{59} & \multirow[t]{5}{*}{$N$} & \multirow[t]{5}{*}{$N$} & \multirow[t]{5}{*}{ CapeOx } & \multirow[t]{5}{*}{ A } & \multirow[t]{5}{*}{130} & \multirow[t]{5}{*}{2} & \multirow[t]{5}{*}{90} & \multirow[t]{5}{*}{6} & 1. Discontinue & \multirow{3}{*}{$\begin{array}{l}\text { 1. Add } \\
\text { loperamid } \\
\text { mg } \\
\text { Sig. } 2 \text { cap } \\
\text { CMT day }\end{array}$} \\
\hline & & & & & & & & & & & \multirow{4}{*}{$\begin{array}{l}\text { 2. Hyoscine-N- } \\
\text { butyl bromide } \\
20 \mathrm{mg} \text { IV STAT } \\
\text { and hydration }\end{array}$} & \\
\hline & & & & & & & & & & & & \\
\hline & & & & & & & & & & & & $\begin{array}{l}\text { 2. Add CPI } \\
\text { mg IV pre }\end{array}$ \\
\hline & & & & & & & & & & & & $\begin{array}{l}\text { 3. Rechallı } \\
\text { with prolo } \\
\text { infusion ti } \\
\text { hours), fin } \\
\text { complete } 1 \\
6 \text { cycles }\end{array}$ \\
\hline \multirow[t]{3}{*}{3} & \multirow[t]{3}{*}{$\mathrm{F}$} & \multirow[t]{3}{*}{60} & \multirow[t]{3}{*}{ Y } & \multirow[t]{3}{*}{$N$} & \multirow[t]{3}{*}{ mFOLFLOX } & \multirow[t]{3}{*}{ A } & \multirow[t]{3}{*}{85} & \multirow[t]{3}{*}{2} & \multirow[t]{3}{*}{20} & \multirow[t]{3}{*}{12} & $\begin{array}{l}\text { 1. Hold and } \\
\text { reduce rate of } \\
\text { administration } \\
\text { to } 80 \mathrm{~mL} / \mathrm{hr}\end{array}$ & \multirow[t]{3}{*}{$\begin{array}{l}12 \text { step } \\
\text { desensitiz } \\
\text { protocol, fi } \\
\text { complete 1 } \\
12 \text { cycles }\end{array}$} \\
\hline & & & & & & & & & & & $\begin{array}{l}\text { 2. CPM } 10 \text { mg, } \\
\text { Dexamethasone } \\
5 \text { mg IV STAT }\end{array}$ & \\
\hline & & & & & & & & & & & $\begin{array}{l}\text { 3. Adrenaline } \\
0.3 \mathrm{mg} \mathrm{IM}\end{array}$ & \\
\hline \multirow[t]{2}{*}{4} & \multirow[t]{2}{*}{$\mathrm{F}$} & \multirow[t]{2}{*}{62} & \multirow[t]{2}{*}{$\mathrm{Y}$} & \multirow[t]{2}{*}{ Y } & \multirow[t]{2}{*}{ FLOX } & $\mathrm{P}$ & 80 & 2 & 120 & 4 & $\begin{array}{l}\text { 1. Adrenaline } 1 \\
\text { mg IM and } \\
\text { hydration }\end{array}$ & $\begin{array}{l}\text { 1. } 12 \text { step } \\
\text { desensitiz } \\
\text { protocol } \mathrm{W} \\
\text { mild HSR } \\
\text { through } \\
\text { desensitiz }\end{array}$ \\
\hline & & & & & & & & & & & & $\begin{array}{l}\text { 2. Patient } \\
\text { requested } \\
\text { discontinu } \\
\text { after the } 4 \\
\text { cycle }\end{array}$ \\
\hline 5 & M & 47 & $\mathrm{~N}$ & $\mathrm{~N}$ & mFOLFLOX & A & 85 & 3 & 110 & 8 & 1. Discontinue & $\begin{array}{l}\text { 1. } 12 \text { step } \\
\text { desensitiz }\end{array}$ \\
\hline & & & & & & & & & & & $\begin{array}{l}2 . \\
\text { Dexamethasone } \\
10 \text { mg IV STAT }\end{array}$ & $\begin{array}{l}\text { protocol, } \mathrm{K} \\
\text { grade-2 Hs } \\
\text { through th } \\
\text { cycle }\end{array}$ \\
\hline & & & & & & & & & & & $\begin{array}{l}\text { 3. Adrenaline } \\
0.5 \mathrm{mg} \mathrm{IM}\end{array}$ & $\begin{array}{l}\text { 2. Discont } \\
\text { after the } 8 \\
\text { cycle }\end{array}$ \\
\hline 6 & M & 45 & Y & $\mathrm{N}$ & CapeOx & A & 100 & 2 & 15 & 3 & 1. Hold & 1. Rechallı \\
\hline & & & & & & & & & & & $\begin{array}{l}\text { 2. Adrenaline } \\
0.3 \text { mcg IM and } \\
\text { hydration }\end{array}$ & $\begin{array}{l}\text { infusion ti } \\
\text { hours), grc } \\
\text { HSR was }\end{array}$ \\
\hline & & & & & & & & & & & $\begin{array}{l}\text { 3. Admit and } \\
\text { rechallenge } \\
\text { oxaliplatin 20- } \\
40 \mathrm{~mL} / \mathrm{hr}\end{array}$ & $\begin{array}{l}\text { discontinu } \\
\text { 2. Skin tes } \\
\text { Positive }\end{array}$ \\
\hline
\end{tabular}




\begin{tabular}{|c|c|c|c|c|c|c|c|c|c|c|c|c|}
\hline No. & Gender & $\begin{array}{l}\text { Age } \\
\text { (years) }\end{array}$ & $\begin{array}{l}\text { Prior } \\
\text { platinum } \\
\text { exposure }\end{array}$ & Metastasis & Regimen & Purpose & $\begin{array}{l}\text { Dose per } \\
\text { BSA } \\
\text { (mg/m2) }\end{array}$ & $\begin{array}{l}\text { Cycle } \\
\text { number } \\
\text { at } \\
\text { event }\end{array}$ & $\begin{array}{l}\text { Onset of } \\
\text { HSR } \\
\text { (minutes) }\end{array}$ & $\begin{array}{l}\text { Total } \\
\text { cycle }\end{array}$ & Treatment & Managem \\
\hline \multirow[t]{5}{*}{7} & \multirow[t]{5}{*}{ M } & \multirow[t]{5}{*}{47} & \multirow[t]{5}{*}{ Y } & \multirow[t]{5}{*}{$\mathrm{N}$} & \multirow[t]{5}{*}{ FLOX } & \multirow[t]{5}{*}{$P$} & \multirow[t]{5}{*}{85} & \multirow[t]{5}{*}{1} & \multirow[t]{5}{*}{90} & \multirow[t]{5}{*}{6} & $\begin{array}{l}\text { 1. Hold and } \\
\text { oxygen therapy }\end{array}$ & $\begin{array}{l}\text { 1. Skin tes } \\
\text { Negative }\end{array}$ \\
\hline & & & & & & & & & & & $\begin{array}{l}\text { 2. Adrenaline } \\
0.5 \mathrm{mg} \text { IM and } \\
\text { Dexamethasone } \\
10 \mathrm{mg} \text { IV }\end{array}$ & $\begin{array}{l}\text { 2. rechalle } \\
\text { (2nd cycle } \\
\text { with prolo } \\
\text { infusion ti } \\
\text { hours), bu } \\
\text { HSR. }\end{array}$ \\
\hline & & & & & & & & & & & & $\begin{array}{l}\text { 2. } 12 \text { step } \\
\text { desensitiz } \\
\text { protocol ( } \\
\text {-6th cycle) }\end{array}$ \\
\hline & & & & & & & & & & & $\begin{array}{l}\text { 3. Admit and } \\
\text { then slow }\end{array}$ & \\
\hline & & & & & & & & & & & $\begin{array}{l}\text { infusion about } \\
4 \text { hours }\end{array}$ & \\
\hline \multirow[t]{4}{*}{8} & \multirow[t]{4}{*}{ M } & \multirow[t]{4}{*}{56} & \multirow[t]{4}{*}{ N } & \multirow[t]{4}{*}{$\mathrm{N}$} & \multirow[t]{4}{*}{ mFOLFLOX } & \multirow[t]{4}{*}{$A$} & \multirow[t]{4}{*}{80} & \multirow[t]{4}{*}{6} & \multirow[t]{4}{*}{80} & \multirow[t]{4}{*}{6} & 1. Hold & \multirow{4}{*}{$\begin{array}{l}\text { Discontint } \\
\text { according } \\
\text { therapeuti } \\
\text { plan }\end{array}$} \\
\hline & & & & & & & & & & & $\begin{array}{l}\text { 2. Symbicort }{ }^{\mathrm{TM}} \\
\text { (160/4.5) } 4 \\
\text { puffs and } \\
\text { oxygen therapy }\end{array}$ & \\
\hline & & & & & & & & & & & $\begin{array}{l}\text { 3. CPM } 10 \mathrm{mg} \\
\text { IV }\end{array}$ & \\
\hline & & & & & & & & & & & $\begin{array}{l}\text { 4. Continue } \\
\text { infusion rate } 80 \\
\mathrm{~mL} / \mathrm{hr}\end{array}$ & \\
\hline 9 & $M$ & 44 & $\mathrm{~N}$ & $\mathrm{~N}$ & CapeOx & $A$ & 90 & 6 & 120 & 6 & $\begin{array}{l}\text { 1. Hyoscine-N- } \\
\text { butyl bromide } \\
20 \mathrm{mg} \text { IV and } \\
\text { CPM } 10 \mathrm{mg} \text { IV } \\
\text { STAT }\end{array}$ & $\begin{array}{l}\text { Discontinı } \\
\text { according } \\
\text { therapeuti } \\
\text { plan }\end{array}$ \\
\hline \multirow[t]{2}{*}{10} & \multirow[t]{2}{*}{$F$} & \multirow[t]{2}{*}{73} & \multirow[t]{2}{*}{ Y } & \multirow[t]{2}{*}{ Y } & \multirow[t]{2}{*}{ CapeOx } & \multirow[t]{2}{*}{$\mathrm{P}$} & \multirow[t]{2}{*}{120} & \multirow[t]{2}{*}{3} & \multirow[t]{2}{*}{120} & 3 & $\begin{array}{l}\text { 1. Loperamide } 2 \\
\text { mg } 2 \text { capsules } \\
\text { STAT }\end{array}$ & \multirow[t]{2}{*}{$\begin{array}{l}\text { Discontint } \\
\text { [Hand foo } \\
\text { syndrome } \\
\text { grade 3] }\end{array}$} \\
\hline & & & & & & & & & & & 2. Hydration & \\
\hline
\end{tabular}

A=Adjuvant, $P=$ Palliative, $C M T=$ chemotherapy

Risk factors for hypersensitivity reaction

To investigate the potential risk factors for the development of oxaliplatin HSR, we initiated logistic regression model, in which the following factors were included: (1) age, (2) gender, (3) metastasis, (4) prior platinum exposure, (5) history of drug hypersensitivity, (6) neutrophil count, (7) lymphocyte count, (8) monocyte count, (9) eosinophil count, (10) serum albumin, (11) serum creatinine, (12) oxaliplatin infusion number, and (13) total dose of oxaliplatin. Though the univariate analysis, in total 8 factors had $p$-values $<0.2$ which met the criteria for inclusion in multivariate analysis (Table 4 ). 
Table 4

Univariate analysis and multivariate analysis for candidate risk factors for oxaliplatin hypersensitivity [ $\mathrm{N}=245]$

\begin{tabular}{|c|c|c|c|c|c|c|c|c|}
\hline Factors & \multicolumn{4}{|c|}{ Univariate analysis } & \multicolumn{4}{|c|}{ Multivariate analysis } \\
\hline Age & 0.005 & 1.005 & $0.982-1.028$ & 0.675 & & & & \\
\hline Gender, male & 0.663 & 1.941 & $1.132-3.326$ & $0.016^{\star}$ & 0.966 & 2.628 & $1.450-4.763$ & 0.001 \\
\hline Metastasis, yes & -0.034 & 0.712 & $0.424-1.196$ & 0.199 & & & & \\
\hline History of drug hypersensitivity, yes & 0.460 & 1.584 & $0.69-3.637$ & 0.278 & & & & \\
\hline Neutrophil count (cell/mm3) & 0.0002 & 0.99998 & $0.9998-1.0002$ & 0.826 & & & & \\
\hline Total lymphocyte count (cell/mm³) & 0.0004 & 0.9996 & 0.9992-0.9999 & $0.037^{\star}$ & & & & \\
\hline Monocyte count (cell/mm³) & -0.001 & 0.999 & $0.998-1.000$ & 0.197 & -0.002 & 0.998 & 0.996-0.999 & 0.007 \\
\hline Serum creatinine $(\mathrm{mg} / \mathrm{dL})$ & 1.128 & 3.090 & $0.984-9.707$ & 0.053 & & & & \\
\hline Oxaliplatin infusion number (times) & 0.028 & 1.028 & $0.929-1.138$ & 0.589 & & & & \\
\hline Total dose of oxaliplatin (mg) & 0.0003 & 1.000 & $1.000-1.001$ & 0.234 & & & & \\
\hline Constant & & & & & 3.419 & & & \\
\hline${ }^{*} p<0.05$ & & & & & & & & \\
\hline
\end{tabular}

After backward stepwise elimination method, 5 final factors were selected: gender, prior platinum exposure, eosinophil count, monocyte count and serum albumin. The odds ratio $(95 \% \mathrm{Cl})$ from the multivariate model was presented in Table 4 . We established the incidence of hypersensitivity in colorectal cancer patients treated with this oxaliplatin base regimen $(\mathrm{Y})$ model:

$Y=3.419+[0.966 x($ Gender; if male $=1)]+[0.818 x$ (if prior platinum exposure) $]+[0.003 x($ number of eosinophil count) $]-[0.002 x($ number of monocyte count) $]$ [1.111x(serum albumin)]

\section{Discussion}

Hypersensitivity reactions to oxaliplatin result in the withdrawal of the chemotherapy or reducing the number of therapeutic options. The incidence of oxaliplatin HSR is growing as a consequence of the increasing use of oxaliplatin in colorectal cancer. The incidence of oxaliplatin HSR in this study was $37.96 \%$, relatively higher than the rates described in previous studies (8.9-23.8\%) $11,4,7,9-11,28]$, suggesting racial effects and dosage effects. The MOSAIC trail, a large, randomized phase 3 trial in Western countries, showed $10.3 \%$ of 1,100 patients who received 5-fluorouracil with oxaliplatin experienced HSR.18 The report from Japanese patients indicated that $22.9 \%$ of 108 patients experienced hypersensitivity.[24] Additionally, the dose of oxaliplatin in the MOSAIC trail was $85 \mathrm{mg}$ per square meter or was reduced to $75 \mathrm{mg}$ per square meter in the event of persistent neuropathy[1], but the average dose of oxaliplatin in this study was $110 \mathrm{mg}$ per square meter. Interestingly, grade 3/4 HSR in this study was similar to the previous study.[1, 24] Ten patients experienced grade 3/4 HSR to oxaliplatin and 6 patients were reintroduced to oxaliplatin. The reintroduction of oxaliplatin was done after skin testing to oxaliplatin or using a 12-step desensitization protocol, developed by the Dana-Farber Cancer Institute and the Brigham and Women's Hospital.[5, 14]

The identification of risk of developing HSR is an important issue for the prevention and related management of serious events. The risk factors to platinum drugs related HSR, especially carboplatin and cisplatin were examined. Only a few studies were focused to identify potential risk factors for oxaliplatin-related HSR.[15] This study emphasized the risk factors to recognize patients at risk of oxaliplatin-related HSR. Logistic regression analysis found five significant factors, three risk factors (male, prior platinum exposure, eosinophil count) and two protective factors (monocyte count, serum albumin).

Okayama et al., reported that male patients and eosinophil count in peripheral blood were independent variables for oxaliplatin-related HSR,[19] which were similar to our finding. Eosinophil, one of the immunologic cells, absolutely characterizes drug HSR especially drug eruptions, consequently in patients with high eosinophil counts, this may be a component in mechanisms of HSR to oxaliplatin.[12, 19] This is contrary to Parel et al., Seki et al., and Kim et al., studies which suggested that female was higher risk of oxaliplatin-related HSR,[10, 21, 24] explained by a possible role of hormonal influences. Several studies have also reported no correlation between gender and HSR.[25, 28] Our study demonstrated that prior platinum exposure was the independent risk factor which was distinguished from previous studies.

On the other hand, we found that lower monocyte count and serum albumin was significantly associated with the incidence of oxaliplatin-related HSR. Seki et al., also suggested that female, preexisting allergies, lower LDH level, higher neutrophil count and lower monocyte count were associated with the incidence of HSR.[24] Serum albumin is commonly utilized as a marker of nutritional status. Malnutrition degrades both the innate and adaptive immune system.[3] Low serum albumin level increases vascular permeability and increases interstitial volume, which potentiates immediate HSR.[27] However, Nishihara et al., reported that serum albumin level above $4.1 \mathrm{~g} / \mathrm{dL}$ was the potential risk associated with the incidence of oxaliplatin-related HSR. Oxaliplatin may also act as a

Page 9/11 
hapten, binding to macromolecular carrier proteins, such as albumin.[17] A reason for the variation of risk factors was the ethnic differences between the participants in this study. Finally, we found that treatment regimen and oxaliplatin dose was not associated with an increased risk of oxaliplatin-related HSR.

This retrospective study was conducted to clarify the risk factors for oxaliplatin-related HSR in Southeast Asia and demonstrated the management of severe oxaliplatin-related HSR in real situation. Limitations of this study include its retrospective design and incomplete clinical data. Some medical records were insufficient, and hypersensitivity symptoms were not actively followed. Further prospective studies are needed to refine this oxaliplatin-related hypersensitivity prediction model to be more precise and develop the final model as a guide to prevent oxaliplatin-related HSR.

\section{Conclusion}

Oxaliplatin-related hypersensitivity is a significant potential adverse reaction. The incidence was approximately $38 \%$, with grade $3 / 4$ events in $4 \%$. In this study, males, prior platinum exposure, eosinophil count, monocyte count, and serum albumin were the independent risk factors which were associated with the incidence of oxaliplatin-related hypersensitivity.

\section{Declarations}

Acknowledgments: None.

Funding: This research did not receive any specific grant from funding agencies in the public, commercial, industry, or not-for-profit sectors.

Conflicts of interest/Competing interests: None to declare

Availability of data and material: The datasets generated during or analysed during the current study are not publicly available due to data policy of Chulalongkorn Memorial Hospital but are available from the corresponding author on reasonable request.

Code availability: IBM ${ }^{\circledR}$ SPSS ${ }^{\circledR}$ version 22 licensed provided by Chulalongkorn University via splm.it.chula.ac.th.

Authors' contributions: All authors contributed to the study conception and design. Material preparation and data collection were performed by Sirinoot Palapinyo. The data analysis was performed by Sirinoot Palapinyo and Nutthada Areepium. The first draft of the manuscript was written by Sirinoot Palapinyo, and all authors commented and edited on previous versions of the manuscript. All authors read and approved the final manuscript. The content has not been published elsewhere.

Ethics approval: This study was approved by the Institutional Review Board (IRB), Faculty of Medicine, Chulalongkorn University (IRB no. 323/61)

Consent to participate: Not applicable

Consent for publication: Not applicable

\section{References}

1. André T, Boni C, Mounedji-Boudiaf L, Navarro M, Tabernero J, Hickish T, Topham C, Zaninelli M, Clingan P, Bridgewater J, Tabah-Fisch I, de Gramont A (2004) Oxaliplatin, fluorouracil, and leucovorin as adjuvant treatment for colon cancer. N Engl J Med 350:2343-2351

2. Benson AB, Venook AP, Al-Hawary MM, Arain MA, Chen Y-J, Ciombor KK, Cohen S, Cooper HS, Deming D, Farkas L, Garrido-Laguna I, Grem JL, Gunn A, Hecht JR, Hoffe S, Hubbard J, Hunt S, Johung KL, Kirilcuk N, Krishnamurthi S, Messersmith WA, Meyerhardt J, Miller ED, Mulcahy MF, Nurkin S, Overman MJ, Parikh A, Patel H, Pedersen K, Saltz L, Schneider C, Shibata D, Skibber JM, Sofocleous CT, Stoffel EM, Stotsky-Himelfarb E, Willett CG, Gregory KM, Gurski LA (2021) Colon Cancer, Version 2.2021, NCCN Clinical Practice Guidelines in Oncology Journal of the National Comprehensive Cancer Network J Natl Compr Canc Netw. 19:329-359

3. Bourke CD, Berkley JA, Prendergast AJ (2016) Immune Dysfunction as a Cause and Consequence of. Malnutrition Trends Immunol 37:386-398

4. Brandi G, Pantaleo MA, Galli C, Falcone A, Antonuzzo A, Mordenti P, Di Marco MC, Biasco G (2003) Hypersensitivity reactions related to oxaliplatin (OHP). Br J Cancer 89:477-481

5. Castells M (2006) Desensitization for drug allergy Current opinion in allergy and clinical immunology. 6:476-481

6. Common Terminology Criteria for Adverse Events (CTCAE) Version 5. Published: November 27.US Department of Health and Human Services NloH, National Cancer Institute

7. Gowda A, Goel R, Berdzik J, Leichman CG, Javle M (2004) Hypersensitivity Reactions to oxaliplatin: incidence and management Oncology. (Williston Park, NY) 18:1671-1675. discussion 1676, 1680, 1683-1674

8. Grothey A, Goldberg RM (2004) A review of oxaliplatin and its clinical use in colorectal cancer Expert opinion on pharmacotherapy. 5:2159-2170

9. Ichikawa Y, Goto A, Hirokawa S, Kijima M, Ishikawa T, Chishima T, Suwa H, Yamamoto H, Yamagishi S, Osada S, Ota M, Fujii S (2009) Allergic reactions to oxaliplatin in a single institute in. Japan Japanese journal of clinical oncology 39:616-620

10. Kim BH, Bradley T, Tai J, Budman DR (2009) Hypersensitivity to Oxaliplatin: An Investigation of Incidence and Risk Factors, and Literature Review Oncology 76: 231-238

11. Kim MY, Kang SY, Lee SY, Yang MS, Kim MH, Song WJ, Kim SH, Kim YJ, Lee KW, Cho SH, Min KU, Lee JS, Kim JH, Chang YS (2012) Hypersensitivity reactions to oxaliplatin: clinical features and risk factors in Koreans. Asian Pacific journal of cancer prevention: APJCP 13:1209-1215 
12. Kuruvilla M, Khan DA (2016) Eosinophilic Drug Allergy Clinical reviews in allergy \& immunology 50:228-239

13. Lee C, Gianos M, Klaustermeyer WB (2009) Diagnosis and management of hypersensitivity reactions related to common cancer chemotherapy agents Annals of Allergy. Asthma \& Immunology 102:179-187

14. Lee CW, Matulonis UA, Castells MC (2005) Rapid inpatient/outpatient desensitization for chemotherapy hypersensitivity: standard protocol effective in 57 patients for 255 courses Gynecologic oncology. 99:393-399

15. Makrilia N, Syrigou E, Kaklamanos I, Manolopoulos L, Saif MW (2010) Hypersensitivity Reactions Associated with Platinum Antineoplastic Agents: A Systematic Review Metal-Based Drugs 2010: 207084

16. Mori Y, Nishimura T, Kitano T, Yoshimura K, Matsumoto S, Kanai M, Hazama M, Ishiguro H, Nagayama S, Yanagihara K, Teramukai S, Chiba T, Sakai Y, Fukushima M (2010) Oxaliplatin-free interval as a risk factor for hypersensitivity reaction among colorectal cancer patients treated with FOLFOX Oncology. 79:136-143

17. Nishihara M, Nishikura K, Morikawa N, Yokoyama S (2017) Factors Influencing the Appearance of Oxaliplatin. Induced Allergy Biological \& pharmaceutical bulletin 40:2105-2109

18. Ohta H, Hayashi T, Murai S, Shiouchi H, Ando Y, Kumazawa S, Ito K, Ikeda Y, Matsuoka H, Maeda K, Kawada K, Yasuda K, Yamada S (2017) Comparison between hypersensitivity reactions to cycles of modified FOLFOX6 and XELOX therapies in patients with colorectal cancer Cancer. Chemotherapy and Pharmacology 79:1021-1029

19. Okayama T, Ishikawa T, Sugatani K, Yoshida N, Kokura S, Matsuda K, Tsukamoto S, Ihara N, Kuriu Y, Nakanishi M, Nakamura T, Kamada K, Katada K, Uchiyama K, Takagi T, Handa O, Konishi H, Yagi N, Naito Y, Otsuji E, Hosoi H, Miki T, Itoh Y (2015) Hypersensitivity Reactions to Oxaliplatin: Identifying the Risk Factors and Judging the Efficacy of a Desensitization Protocol Clinical therapeutics. 37:1259-1269

20. Otani IM, Wong J, Banerji A (2017) Platinum Chemotherapy Hypersensitivity: Prevalence and Management Immunology and allergy clinics of North America. 37:663-677

21. Parel M, Ranchon F, Nosbaum A, You B, Vantard N, Schwiertz V, Gourc C, Gauthier N, Guedat M-G, He S, Kiouris E, Alloux C, Vial T, Trillet-Lenoir V, Freyer G, Berard F, Rioufol C (2014) Hypersensitivity to oxaliplatin: clinical features and risk factors. BMC Pharmacol Toxicol 15:1-1

22. Rawla P, Sunkara T, Barsouk A (2019) Epidemiology of colorectal cancer: incidence, mortality, survival, and risk factors Prz Gastroenterol. 14:89-103

23. Raymond E, Faivre S, Woynarowski JM, Chaney SG (1998) Oxaliplatin: mechanism of action and antineoplastic activity. Semin Oncol 25:4-12

24. Seki K, Senzaki K, Tsuduki Y, loroi T, Fujii M, Yamauchi H, Shiraishi Y, Nakata I, Nishiguchi K, Matsubayashi T, Takakubo Y, Okamura N, Yamamori M, Tamura T, Sakaeda T (2011) Risk factors for oxaliplatin-induced hypersensitivity reactions in Japanese patients with advanced colorectal cancer. Int J Med Sci 8:210-215

25. Shibata Y, Ariyama H, Baba E, Takii Y, Esaki T, Mitsugi K, Tsuchiya T, Kusaba H, Akashi K, Nakano S (2009) Oxaliplatin-induced allergic reaction in patients with colorectal cancer in Japan. Int J Clin Oncol 14:397-401

26. Siu SW, Chan RT, Au GK (2006) Hypersensitivity reactions to oxaliplatin: experience in a single institute Annals of oncology: official. journal of the European Society for Medical Oncology 17:259-261

27. Soeters PB, Wolfe RR, Shenkin A (2019) Hypoalbuminemia: Pathogenesis and Clinical Significance. Journal of Parenteral and Enteral Nutrition 43:181193

28. Yamauchi H, Goto T, Takayoshi K, Sagara K, Uoi M, Kawanabe C, Matsunaga M, Miyoshi T, Uchino K, Misumi N, Nishino T (2015) A retrospective analysis of the risk factors for allergic reactions induced by the administration of oxaliplatin. Eur $\mathrm{J}$ Cancer Care 24:111-116

29. Zhao Y, An X, Xiang XJ, Feng F, Wang FH, Wang ZQ, Xu RH, He YJ, Li YH (2010) [Clinical features of hypersensitivity reactions to oxaliplatin among Chinese colorectal cancer patients]. Chinese journal of cancer 29:102-105 